\title{
Correlação entre Hiperglicemia e Células do SNC, com Enfoque na Atividade Glial
}

\author{
Correlation Between Hyperglicemia and CNS Cells, Focusing on the Glial Activity
}

Amanda de Souza Mello ${ }^{1}$,André Quincozes-Santos², Cláudia Funchal ${ }^{3}$

\begin{abstract}
RESUMO
Introduçáo. Entre os mecanismos biológicos que originam o quadro hiperglicêmico a predominância é do diabetes melittus (DM). O DM representa um grupo de desordens metabólicas caracterizadas por hiperglicemia crônica que ocasiona severas alteraçôes celulares e teciduais. Objetivo. O presente trabalho analisou através de revisão da literatura o comportamento de células gliais expostas a elevadas concentraçóes de glicose, similares às observadas no DM. Método. Foi realizada uma revisão literária através de artigos científicos das bases de dados Pubmed, Science Direct, Scopus e Scielo. Resultados. Foram selecionados artigos e livros entre 1988 e 2009 que discutiam hiperglicemia, sistema nervoso central e que relacionavam hiperglicemia e células gliais. Conclusáo. A hiperglicemia crônica proporcionada pelo DM pode influenciar de maneira danosa o metabolismo cerebral exercendo açôes sobre a atividade glial. Podendo afetar a sobrevivência neuronal através da excitotoxicidade glutamatérgica e da produção de espécies reativas de oxigênio (ERO) e de espécies reativas de nitrogênio (ERN) que geram como consequência o processo de neuroinflamação. Tal processo inflamatório pode resultar em dano e morte neural caracterizando um processo neurodegerativo.
\end{abstract}

Unitermos. Hiperglicemia, Astrócitos, Glutamato, Excitoxicidade, Estresse Oxidativo.

Citaçáo. Mello AS, Quincozes-Santos A, Funchal C. Correlação entre Hiperglicemia e Células do SNC, com Enfoque na Atividade Glial.

\begin{abstract}
Introduction. Among the biological mechanisms that originate the hyperglycemic state the prevalence is the diabetes mellitus (DM). DM represents a group of metabolic disorders characterized by chronic hyperglycemia that causes severe cellular and tissue alterations. Objective. This study examined through literature review the actions of glial cells exposed to high glucose concentrations similar to those observed in DM. Method. We performed a literature review of scientific articles through Pubmed, Science Direct, Scopus and Scielo. Results. We selected articles and books between 1988 and 2009 that discussed hyperglycemia, central nervous system, and that related hyperglycemia and glial cells. Conclusion. The chronic hyperglycemia provided by DM may harmful influence brain metabolism exerting actions on the glial activity. It may affect neuronal survival by glutamatergic excitotoxicity and the production of reactive oxygen species (ROS) and nitrogen reactive species (RNS) that generates as a consequence the process of neuroinflammation. The inflammatory process can result in damage and death neural characterizing a neurodegenerative process.
\end{abstract}

Keywords. Hyperglycemia, Astrocytes, Glutamate, Excitotoxicity, Oxidative Stress.

Citation. Mello AS, Quincozes-Santos A, Funchal C. Correlation Between Hyperglicemia and CNS Cells, Focusing on the Glial Activity.

\footnotetext{
Trabalho realizado no Centro Universitário Metodista do IPA, Porto AlegreRS, Brasil.

1. Farmacêutica, Mestranda do Programa de Pós-Graduação em Biociências e Reabilitaçáo do Centro Universitário Metodista do IPA, Porto Alegre-RS, Brasil.

2. Farmacêutico Bioquímico, Doutor em Bioquímica pela Universidade Federal do Rio Grande do Sul (UFRGS), Porto Alegre-RS, Brasil.

3. Farmacêutica Bioquímica, Doutora em Bioquímica pela Universidade Federal do Rio Grande do Sul (UFRGS), Docente do Programa de Pós-Graduaçáa em Biociências e Reabilitaçáo do Centro Universitário Metodista do IPA, Porto Alegre-RS, Brasil.
}

Endereço para correspondência: Dra. Cláudia Funchal Rede Metodista de Educação do Sul Centro Universitário Metodista do IPA Rua Cel. Joaquim Pedro Salgado, 80 Tel: 555133161233

CEP 90420-060, Porto Alegre-RS, Brasil. E-mail: claudia.funchal@metodistadosul.edu.br; csfunchal@yahoo.com.br 


\section{INTRODUÇÃO}

O equilíbrio do metabolismo corporal exige um elevado ajuste fino entre açóes desencadeadas pelo sistema nervoso central (SNC) e pelo sistema endócrino. Uma das funçôes chave para manutenção da homeostase é assegurar o controle dos níveis de glicose no plasma, onde o principal responsável por este controle é o pâncreas, mais precisamente as células beta através da liberação do hormônio insulina. Quando este equilíbrio é rompido, ocorre aumento da concentração de glicose no plasma, caracterizando um quadro de hiperglicemia, cujas consequências neurológicas, embora não totalmente esclarecidas, podem influenciar largamente o metabolismo cerebral ${ }^{1,2}$.

$\mathrm{O}$ diabetes melittus (DM) é caracterizado como um quadro de hiperglicemia crônica. O DM é apontado hoje como um dos maiores problemas de saúde pública. Sua incidência e prevalência estão aumentando e alcançando proporções epidêmicas. Estima-se que em 2030, aproximadamente, 366 milhóes de pessoas serão portadoras de algum tipo de DM. As alteraçōes metabólicas causadas pela hiperglicemia são responsáveis pelo desenvolvimento de complicaçóes crônicas importantes que variam de acordo com o tipo e a função celular. Entre as estruturas mais susceptíveis à ação da hiperglicemia estão os vasos sanguíneos (arteroesclerose, microangiopatia) e os neurônios (neuropatia) $)^{1,3}$.

O SNC é considerado um sistema glicodependente, onde a glicose exerce papel fundamental no seu funcionamento. Classicamente o neurônio é considerado a unidade funcional do sistema nervoso, operando em conjunto, formando os chamados circuitos ou redes neuronais, os quais são os responsáveis pelo correto funcionamento de nossas atividades diárias. Outro importante componente desse circuito é a glia, a qual possui atividade extremamente importante e influencia diretamente a atividade neuronal ${ }^{4,5}$.

Hoje, se conhecem no SNC três grandes grupos de células gliais: (1) astrócitos e oligodendrócitos, ambos de origem ectodérmica, que formam a macroglia; (2) a microglia, de origem mesodérmica; (3) células ependimais, de origem ectodérmica ${ }^{6}$.

Os astrócitos constituem aproximadamente 50\% do número total de células do SNC. Eles são divididos em dois tipos: os protoplasmáticos, na substância cinzenta (frequentemente ramificados e com largas expansóes) e os fibrosos, na substância branca (com menos ramificaçóes, cilíndricas e longas) ${ }^{6}$.

Sabe-se que os astrócitos e neurônios apresentam um sofisticado sistema de comunicação recíproca regulando a liberação de neurotransmissores, a excitabilidade neuronal e a transmissão sináptica7 ${ }^{7}$.

O glutamato é o principal neurotransmissor excitatório do SNC. Os astrócitos apresentam entre suas principais funçôes a captação de glutamato, que mantém os níveis de glutamato extracelular. Evidências sugerem que astrócitos expostos a altas concentraçôes de glicose diminuem consideravelmente o imunoconteúdo e secreção da proteína $\mathrm{S} 100 \mathrm{~B}$. A exposição dos neurônios a altas concentraçóes de glutamato e ação deste sobre seus receptores leva ao aumento do influxo de $\mathrm{Ca}^{+2}$ intracelular, evento ao qual se atribui papel decisivo na cascata de alteraçôes que levam à excitotoxicidade $\mathrm{e}^{3,8-15}$.

A produção de espécies reativas do oxigênio (ROS) e espécies reativas do nitrogênio (RNS) parece estar envolvida com a excitoxicidade glutamatérgica. $\mathrm{O}$ estresse oxidativo decorrente do acúmulo de ROS e RNS é um fator essencial associado à morte celular uma vez que podem reagir e oxidar componentes celulares como, lipídios, proteínas e $\mathrm{DNA}^{16-18}$.

\section{MÉTODO}

Foi realizada uma extensa revisão da literatura e a consulta baseou-se em bases banco de dados de artigos científicos como Pubmed, Science Direct, Scopus e Scielo. A busca de artigos nestas bases de dados foi limitada na língua inglesa e portuguesa e os artigos analisados foram selecionados por apresentarem grande pertinência ao tema. Também foram utilizados livros, dissertaçôes de mestrado e teses de doutorado. Foram selecionados artigos e livros entre 1988 e 2009 que discutiam hiperglicemia, SNC e que relacionavam hiperglicemia e células gliais.

\section{RESULTADOS}

Alguns estudos mostram que astrócitos em presença de elevado conteúdo de glicose reduzem consideravelmente o imunoconteúdo e a secreção da proteína S100B. Além disso, há uma tendência a diminuição de captação de glutamato, o que pode contribuir para o desenvolvi- 
mento da excitoxicidade, que está relacionada com o aumento da excitabilidade neuronal com hiperativaçáo dos receptores glutamatérgicos e influxo excessivo de $\mathrm{Ca}^{2+}$. O dano e morte neuronal podem ocorrer devido ao aumento do influxo de $\mathrm{Ca}^{2+}$ intracelular e ativaçấo de diversas proteínas, como proteases, fosfatases e fosfolipases, fazendo com que haja um aumento considerável da produção de espécies reativas de oxigênio e nitrogênio originando um quadro de estresse oxidativo sobre as células neurais.

\section{DISCUSSÃO}

\section{Glia e Expressão de Proteínas}

As células gliais eram conhecidas como "glue cells" (células conectivas), assim chamadas em razão de seu conhecido efeito de sustentação e compactação do SNC. Atualmente, sabe-se que além de suas funções de suporte aos neurônios, as células gliais (astrócitos, oligodendrócitos, microglia e células ependimais) podem alterar diretamente a comunicação entre os mesmos, pois estão em contato próximo com a rede neuronal, adquirindo funções como: captação e liberação de neurotransmissores, controle do metabolismo iônico e produção de fatores neurotróficos (Tabela 1) 15,19-22.

Os astrócitos são reconhecidamente importantes na transmissão sináptica e plasticidade neural. Eles são responsáveis pela captação e/ou redistribuição do $\mathrm{K}^{+}$durante a atividade neuronal, detoxificação da amônia, remoção do glutamato e do GABA das sinapses (Tabela 1). Os astrócitos e a microglia são ambos mediadores chave do SNC, em virtude disso podem estar intimamente envolvidos com a excitabilidade neuronal ${ }^{15,21-24}$.

Dentre as proteínas expressas por astrócitos, a proteína glial fibrilar ácida (GFAP) é a maior proteína de filamentos intermediários encontrada no citoplasma de astrócitos e é um marcador de diferenciação astrocitária. Além da GFAP, os astrócitos expressam outros dois tipos de filamentos intermediários: vimentina e nestina. Estes filamentos são as principais proteínas expressas em astrócitos imaturos, enquanto astrócitos em processo de diferenciação ou diferenciados expressam vimentina e GFAP, que formam polímeros entre si. Outra proteína importante expressa e secretada por astrócitos é a proteína ligante de $\mathrm{Ca}^{2+} \mathrm{S} 100 \mathrm{~B}$. Esta é conhecida por promover sobrevivência e diferenciação neural, regular a fosforilação de proteínas alvo e atividade enzimática ${ }^{19,22,25-32}$.

Dados in vitro apontam que após sua liberação pelo astrócito para o meio extracelular, os efeitos da S100B podem ser tróficos ou tóxicos de acordo com sua quantidade extracelular local. Concentraçóes nanomolares parecem exercer efeitos neuroprotetores, aumentando a viabilidade neuronal e estimulando o crescimento neurítico, enquanto concentraçóes micromolares de S100B produzem efeitos neurotóxicos ${ }^{10,32-35}$.

Pouco se sabe sobre os mecanismos envolvidos na secreção de S100B para o meio extracelular. Alguns trabalhos mostram que estímulos sob o comando de estresse metabólico como a hiperglicemia, ocasionam diminuição de $S 100 B$ pelos astrócitos ${ }^{32,36,37}$.

\section{Captaçáo de Glutamato}

O glutamato é o principal neurotransmissor excitatório do SNC. Ele está envolvido em funçôes cognitivas fundamentais como memória, aprendizado e plasticidade sináptica. A síntese e a degradação do glutamato é compartimentalizada entre neurônios e células gliais. No SNC, a glutamina é liberada pelo astrócito e captada pelo

Tabela 1

Principais funçôes dos astrócitos

\begin{tabular}{l|l}
\hline \multicolumn{2}{c}{ Principais funçóes dos astrócitos } \\
\hline Homeostase de íons extracelulares & Metabolismo de neurotransmissores \\
Participação na resposta a injúrias & Participação na resposta imune \\
Formação e manutenção da barreira hematoencefálica & Regulação do espaço extracelular \\
Suporte metabólico através do ciclo glutamato-glutamina e & $\begin{array}{l}\text { Modulação na migração de neurônios nos estágios iniciais do } \\
\text { do metabolismo da glicose e glicogênio }\end{array}$ \\
Síntese e liberação de fatores tróficos e substâncias neuroativas & Progenitores neurais em zonas neurogênicas \\
\hline
\end{tabular}


neurônio e convertida a glutamato pela enzima glutaminase, o qual é liberado na fenda sináptica. A oferta deste neurotransmissor na fenda sináptica é controlada pelas células gliais, especificamente os astrócitos, através da captação de glutamato e enzima glutamina sintetase $\mathrm{e}^{11,38,39}$.

\section{Consequências do Elevado Conteúdo de Glicose para Funçóes Gliais}

Um ambiente com elevada concentração de glicose, promove alteraçóes nas células gliais. Tais mudanças na glia contribuem para a fisiopatologia das desordens do SNC observadas na hiperglicemia e podem ser devido a um efeito direto da glicose, não envolvendo necessariamente um déficit de insulina ${ }^{40}$.

Pesquisadores ${ }^{40}$ mostraram alteraçóes bioquímicas e morfológicas em astrócitos e células de glioma C6 cultivados em meio com alto conteúdo de glicose (12 mM), mimetizando uma condição hiperglicêmica. As células cultivadas em meio com alto conteúdo de glicose apresentaram fenótipos atípicos e heterogêneos e uma acentuada redução do conteúdo de glutationa (cerca de 60\% em astrócitos). Também foi observada uma significativa redução no imunoconteúdo de GFAP (70\% em astrócitos e 40\% em glioma C6) e de S100B (68\% em astrócitos e $40 \%$ em glioma C6). Os astrócitos apresentaram uma redução na secreção de S100B (ao redor de 50\%), porém células de glioma C6 apresentaram aumento na secreção de S100B.

Baseado em estudos realizados in vitro, os dados sugerem que os níveis elevados e crônicos de glicose afetam a atividade astrócitica, pois há uma redução da proteína S100B e de glutationa, o que poderia sugerir o envolvimento do glutamato, pois a glutationa é um dos destinos do glutamato nos astrócitos e a principal defesa antioxidante destas células. Alterações nos níveis de glutamato extracelular podem levar a excitotoxicidade neuronal ${ }^{11,12,40,41}$.

\section{Aumentos dos Níveis de Glutamato e Excitotoxicidade}

Recentemente, estudos têm relacionado alguns distúrbios psiquiátricos (Esquizofrenia, Depressão Maior e Transtorno Bipolar) e doenças neurodegenerativas (Alzheimer, Esclerose Amiotrófica Lateral e Parkinson) com alteraçôes periféricas e centrais na expressão e sensibilidade dos receptores glutamatérgicos ${ }^{11,39}$.
Sabe-se que em condições normais, a neurotransmissão glutamatérgica é delicadamente controlada como um mecanismo de proteção contra a excitotoxicidade, uma vez que a excessiva ativação dos receptores de glutamato está envolvida em várias desordens neurodegenerativas e pode levar à injúria e à morte celular ${ }^{11,42,43}$.

Acredita-se que o glutamato liberado ativa inicialmente os receptores AMPA (ácido 2-amino-3hidroxi-5-metil-4- isoxazolproprionato) e cainato que se colocalizam e que apesar da baixa afinidade relativa pelo glutamato, promoveriam a rápida despolarização da célula através da entrada de íons $\mathrm{Na}^{+}$e $\mathrm{Ca}^{2+}$. A despolarização parcial da membrana plasmática removeria o bloqueio exercido pelo íon $\mathrm{Mg}^{2+}$ do interior do canal do receptor NMDA (N-metil-D-aspartato) ${ }^{11,42,43}$.

A concentração de glutamato nos neurônios glutamatérgicos é menor no meio extracelular (aproximadamente $0,6 \mathrm{mmol} / \mathrm{l}$ ) em comparação com o intracelular (aproximadamente $10 \mathrm{mmol} / \mathrm{l}$ ). Quando os níveis de glutamato extracelular aumentam, ou seja, concentraçóes maiores que $1 \mathrm{mmol} / \mathrm{l}$, ocorrem danos neuronais devido ao aumento da excitabilidade celular em consequência do excessivo influxo de $\mathrm{Ca}^{2+}$ na célula neuronal. Embora o $\mathrm{Ca}^{2+}$ seja fundamental para a homeostase, seu aumento pode levar a morte celular ${ }^{11,17,42-44}$.

O termo "excitotoxicidade" foi descrito por Olney em 1978, quando documentou que a toxicidade do glutamato era devida a sua interação com receptores que mediavam o efeito excitatório nos neurônios. A excitotoxicidade foi relacionada à neuropatologia de várias desordens neurodegenerativas agudas e crônicas. Atualmente, tem se estimado que a excitotoxicidade neuronal possa ser um importante processo na morte celular progressiva ${ }^{42,43,45-47}$.

A excitoxicidade através do influxo excessivo de $\mathrm{Ca}^{+2}$ na célula neuronal proporciona a produção e a liberação do óxido nítrico e a liberação de prostaglandinas pelas enzimas ciclooxigenases. Estes elementos amplificam a excitabilidade celular. $\mathrm{O}$ excesso de $\mathrm{Ca}^{2+}$ intracelular também ativa diversas enzimas, como proteases (ex.: protease citosólica - calpaína), quinase C, fosfatases, fosfolipases (ex.: fosfolipase $\mathrm{A}_{2}$, que ataca a membrana celular e organelas), óxido nítrico sintase neuronal (NOSn), xantina oxidase, e principalmente leva ao aumento da produção de $\operatorname{ROS}^{15,17,46,47}$. 
As espécies produzidas como consequência das enzimas $\mathrm{Ca}^{2+}$ dependentes, como a fosfolipase $\mathrm{A}_{2}$, óxido nítrico sintase (NOS), xantina peroxidase e também a disfunção mitocondrial parecem estar envolvidas com o dano excitotóxico provocado pelo glutamato, podendo representar uma contribuiçáo importante nos mecanismos de degeneração causada por exposição a níveis tóxicos de glutamato ${ }^{47-50}$.

\section{Estresse Oxidativo}

O desequilíbrio entre a formação e a remoção das ROS e RNS no organismo, decorrente da diminuição dos antioxidantes endógenos ou do aumento da geração de espécies oxidantes gera um estado pró-oxidante favorecido em relação ao estado antioxidante chamado estresse oxidativo. Em razão disso ocorre oxidação de macromoléculas e estruturas celulares, podendo resultar em morte celular ${ }^{51}$.

\section{Excitotoxicidade e o Estresse Oxidativo nas Células Neurais}

ROS e RNS em baixas concentraçôes podem auxiliar o organismo na defesa contra patógenos e estão envolvidos em sistemas de sinalização celular. A hiperglicemia está associada ao estresse oxidativo, com consequente aumento de ROS e RNS $S^{2,17,34,52-54}$.

A excitotoxicidade é uma das principais causas de morte celular, e está relacionada a produção de ROS e RNS. Elas podem reagir com as principais classes de biomoléculas, sendo os lipídeos os mais suscetíveis. Também podem danificar a função biológica de proteínas, causando a degradação a peptídeos e eventualmente, a seus respectivos aminoácidos. Os ácidos graxos poliinsaturados, constituintes das membranas biológicas são também um alvo importante para o ataque de ROS. A oxidação destes lipídeos é conhecida como peroxidação lipídica, um processo que leva a alteraçôes estruturais e funcionais das membranas celulares e intracelulares, prejudicando seu metabolismo, podendo inclusive induzir a morte celular $2,17,51,53-55$.

\section{Neuroinflamaçáo e Dano Celular}

O cérebro é particularmente susceptível ao efeito das ROS e RNS devido a seu alto consumo de oxigênio, presença de lipídios e baixas defesas antioxidantes em relação a outros órgãos. As células neuronais podem produzir, dependendo do quadro clínico, substâncias nocivas à célula, tais como citocinas pró-inflamatórias, e prostaglandinas. Citocinas são componentes críticos do processo inflamatório implicadas na morte de oligodendrócitos, degeneração axonal, disfunção neuronal e na patogênese da neuroinflamação $0^{50,56-60}$.

As células endoteliais e gliais, uma vez ativadas por citocinas pró-inflamatórias, liberam quimiocinas. As quimiocinas são responsáveis pela movimentação dos leucócitos, incluindo sua migração para locais de inflamação tecidual a partir do sangue. As quimiocinas liberadas favorecem a migração de células como neutrófilos, linfócitos T e monócitos. Quando essas células atingem o parênquima cerebral, secretam mais citocinas e quimiocinas, amplificando o processo inflamatório ${ }^{61-63}$.

Além da participação das citocinas e quimiocinas, existe ainda a presença de enzimas proteolíticas que degradam a membrana basal e a matriz extracelular (ECM) no processo de migração das células ativadas para o SNC. As enzimas metaloproteinases (MMPs) facilitam a infiltração das células imunes no parênquima cerebral e à ação de citocinas pró-inflamatórias associadas à membrana, como a clivagem do TNF-alfa da superfície celular amplificando sua atividade mielinotóxica ${ }^{15,64}$.

As MMPs constituem uma família de endopeptidases zinco-dependentes, secretadas por vários tipos celulares como macrófagos e fibroblastos capazes de degradar componentes do tecido conjuntivo e da membrana basal. Estas enzimas participam normalmente dos processos fisiológicos, como desenvolvimento fetal, angiogênese, remodelamento do tecido e no processo de migraçáo de células inflamatórias. Contudo, durante processos patológicos, como neuroinflamação, a produção aumentada de MMP proporciona degradação da matriz extracelular e amplificação da lesão tecidual ${ }^{2,15,46,56}$.

As açóes neurodegenerativas decorrentes de quadros hiperglicêmicos são associadas geralmente com as respostas inflamatórias crônicas, onde estas poderão ser geradas a partir de ROS. O quadro hiperglicêmico está associado ao estresse oxidativo e pode implicar em morte celular $^{2,15,56}$. 


\section{CONCLUSÃO}

Independentemente de sua etiologia, o DM é considerado um grupo de desordens metabólicas caracterizada por hiperglicemia crônica, a qual pode levar a sérias disfunçôes teciduais, que variam de acordo com o tipo celular e a sua função. A hiperglicemia pode influenciar de maneira danosa o metabolismo cerebral exercendo ações sobre os astrócitos. Os efeitos da hiperglicemia levam a uma modulação no imunoconteúdo e na secreção da proteína $\mathrm{S} 100 \mathrm{~B}$, uma importante proteína astrocítica, assim como danos a outros parâmetros gliais como aumento da concentração de glutamato extracelular e redução dos níveis de glutationa. Estímulos excitotóxicos, como alta concentração de glutamato, podem ser um dos fatores responsáveis pela morte neuronal resultante também da ação de ROS e RNS (Tabela 2).

Tabela 2

Principais alteraçōes de células gliais durante a hiperglicemia

\begin{tabular}{c} 
Principais alteraçóes de células gliais durante a hiperglicemia \\
\hline Redução do conteúdo de glutationa \\
Redução no imunoconteúdo de GFAP \\
Modulação no imunoconteúdo de S100B \\
Liberação de quimiocinas \\
Alteração no metabolismo de glutamato \\
Produção de espécies reativas
\end{tabular}

Níveis crônicos de glicose elevada afetam a atividade glial e, portanto, a sobrevivência neuronal através da açấo de ROS e RNS com consequente processo inflamatório. Tal processo pode resultar em dano e morte neural caracterizando um processo neurodegerativo. In vitro, as alterações específicas na atividade dos astrócitos podem ser devidas ao efeito do meio com elevada concentração de glicose.

Portanto, indivíduos com quadro hiperglicêmico crônico podem apresentar uma série de complicaçóes como aumento da frequência e da severidade de patologias do SNC, a maioria delas relacionadas ao aumento da produção de ROS e RNS bem como a neuroinflamaçáo e consequentemente morte celular.

Enfim, à medida que as pesquisas sobre os mecanismos do processo de neurodegeneração evoluem, torna-se cada vez mais evidente o envolvimento de uma rede intrínseca de vias de sinalização, diferentes mecanismos de morte celular e mecanismos proteolíticos relacionados a esse processo.

\section{REFERÊNCIAS}

1.Grassiolli S. Contribuição da função da célula beta pancreática na manutenção da normoglicemia em ratos obesos (Tese). Maringá: UEM, 2004, 162p.

2.Praticò D. Alzheimer's disease and oxygen radicals: new insights. Biochem Pharmacol 2002;63:563-7.

http://dx.doi.org/10.1016/S0006-2952(01)00919-4

3.Nardin P. Avaliação dos parâmetros bioquímicos e morfológicos em células gliais expostas ao um meio com alto conteúdo de glicose. (Dissertação). Porto Alegre: UFRGS, 2006, 53p.

4.Gusatti M. Modelagem matemática do acoplamento entre atividade elétrica cerebral, metabolismo e hemodinâmica. (Dissertação). Florianópolis: UFSC, 2006, 01p.

5.Jacques-Silva MC, Gemelli T, Carvalho CAS, Funchal C. Papel dos astrócitos no sistema nervoso central. Ciência em Movimento 2007;18:61-9.

6.Young JK, Garvey JS, Huang PC. Glial immunoreactivity for metallothionein in the rat brain. Glia 1991;4:602-10.

http://dx.doi.org/10.1002/glia.440040607

7.Fellin T, Carmignoto G. Neurone-to-astrocyte signalling in the brain represents a distinct multifunctional unit. Physiology in press 2004;559:3-15.

8.Lent R. Cem bilhôes de neurônios: conceitos fundamentais de neurociência. São Paulo: Atheneu, 2001, 714p.

9.Markiewicz I, Lukomska B. The role of astrocytes in the physiology and pathology of the central nervous system. Acta Neurobiol 2006;66:343-58.

10.Maragaskis NJ, Rothstein JD. Glutamate transporters in neurologic disease. Arch Neurol 2001;58:365-70.

http://dx.doi.org/10.1001/archneur.58.3.365

11.Maragakis NJ, Rothstein JD. Glutamate transporters: animal models to neurologic disease. Neurobiol Dis 2004;15:461-73.

http://dx.doi.org/10.1016/j.nbd.2003.12.007

12.Puskas F, Grocott HP, White WD, Mathew JP, Newman M, Bar-Yosef S. Intraoperative hyperglycemia and cognitive decline after CABG. Ann Thorac Surg 2007;84:1467-73.

http://dx.doi.org/10.1016/j.athoracsur.2007.06.023

13.Choi DW. Calcium-mediated neurotoxity: relationship to specific channel types and role in ischemic damage. Trends in Neurosci 1988A;11:465-9.

http://dx.doi.org/10.1016/0166-2236(88)90200-7

14.Choi DW. Glutamate neurotoxicity and diseases of the nervous system. Neuron 1988B;1:623-34.

http://dx.doi.org/10.1016/0896-6273(88)90162-6

15.Milligan ED, Watkins LR. Pathological and protective roles of glia in chronic pain. Nature 2009; 10:1-14.

16.Trotti D, Rossi D, Gjesdal O, Levy LM, Racagni G, Danbolt NC, Volterra, A. Peroxynitrite inhibits glutamate transporter subtypes. J Biol Chem 1996;5976-79.

17.Emerit J. Neurodegenerative disease and oxidative stress. Biomed Pharmacother 2004;58:39-46.

http://dx.doi.org/10.1016/j.biopha.2003.11.004

18.Sanz A, Caro P, Ibanez J, Gomez J, Gredilla R, Barja G. Dietary restriction at old age lowers mitochondrial oxygen radical production and leak at complex 
I and oxidative DNA damage in rat brain. J Bioenerg 2005;37:83-90.

http://dx.doi.org/10.1007/s10863-005-4131-0

19.Kimelberg H K, Noremberg M D. Astrocytes. Scientific Am 1989;260:44-52. http://dx.doi.org/10.1038/scientificamerican0489-66

20.Rakic P. Elusive radial glial cells: historical and evolutionsary perspective. Glia 2003;43:19-32.

http://dx.doi.org/10.1002/glia.10244

21.Nedeegaard M, Ranson B, Goldman SA. New roles for astrocytes: Redefining the functional architecture of the brain. Trends Neurosci 2003;26:523-30. http://dx.doi.org/10.1016/j.tins.2003.08.008

22.Chenn A. A Top-NOTCH Way to Make Astrocytes, Dev Cell 2009;1:1-2. 23.Anderson CM, swanson RA Astrocyte glutamate transport: review of properties, regulation and physiological functions. Glia 2000;32:1-14.

http://dx.doi.org/10.1002/1098-1136(200010)32:1<1::AIDGLIA10>3.0.CO;2-W

http://dx.doi.org/10.1002/1098-1136(200010)32:1<1::AIDGLIA10>3.3.CO;2-N

24. Waaagepetersen HS, Sonnewald U, Schoesboe A. Compartmentation of glutamine, glutamate, and GABA metabolism in neurons and astrocytes: functional implications. Neuroscientist 2003;9:398-403.

http://dx.doi.org/10.1177/1073858403254006

25.Lefrançois T, Peschannski M, Tardy M. Neuritic ougrowth associated with astroglial phenotypic changers induced by antisense glial fibrillary acidic protein (GFAP) mRNA in jured neuron-astrocyte cocultures. J Neurosci 1997;17:4121-8.

26. Menet V, Ribota M G, Chauvet N, Crian M J, Colucciciguyon E, Privat A. Inactivation of the glial fibrillary acidic protein gene, but not of vimentin, improves neuronal surnical and neurite growth by modifying adhesion molecule espression. J Neurosci 2001;16:6147-58.

27.Eng LF, Ghirnikar RS. GFAP and astrogliosis. Brain Pathol 1994;4:229-37. http://dx.doi.org/10.1111/j.1750-3639.1994.tb00838.x

28.Clark EJ, Allan V. Intermediate filaments: vimentin moves in. Curr Biol 2002;12:596-8

http://dx.doi.org/10.1016/S0960-9822(02)01102-8

29.Weinstein DE, Shelannski ML, Liem RK. Suppression by antisense mRNA demostrates a requirement for the glial fibrillary acidic protein in the formation of stable astrocytic processes in response to neurons. J Cell Biol 1991;112:1205-13

\section{http://dx.doi.org/10.1083/jcb.112.6.1205}

30.Alexanian AR, Burgurg JR. Neuronal survival activity of S100B beteba is enhacend by calcineurin inhibitors and requires activation of nf-kappab. FASEB J 1999;13:1611-20.

31.Rothermundt M, Peter M, Prehn HM, Arolt V. S100b in brain damage and neurodegeneration. Microsc Res Tech 2003;60:614-32.

http://dx.doi.org/10.1002/jemt.10303

32.Sedaghat F, Notopoulos A. S100 protein family and its application in clinical practice. Hippokratia 2008;12:198-204.

33.Li Y, Wang J, Sheng, J G, Lui L, Barger S W, Jones R A, et al. S100B Increases of b-amyloid precursor protein and its encoding mRNA in rat neuronal cultures. J Neurochem 1998;71:1421-8.

http://dx.doi.org/10.1046/j.1471-4159.1998.71041421.x

34.Tramontina F, Tramontina AC, Souza DF, Leite MC, Gottfried C, Souza DO, et al. Glutamate uptake is stimulated by extracellular S100B in hippocampal astrocytes. Cell Mol Neurobiol 2006;26:81-6.

35.Nardin P, Tortorelli L, Quincozes-Santos A, Almeida LM, Leite MC, Thomazi AP, et al. S100B Secretion in Acute Brain Slices: Modulation by Extracellular Levels of $\mathrm{Ca}(2+)$ and $\mathrm{K}(+)$. Neurochem Res 2009;15:1-2.

36.Tramontina F, Leite MC, Gonçalves D, Tramontina AC, Souza DF, Frizzo
JK, et al. High glutamate decreases S100B secretion by a mechanism dependent on the glutamate transporter. Neurochem Res 2006;31:815-20.

http://dx.doi.org/10.1007/s11064-006-9085-z

37.Gerlach R, Demel G, Konig HG, Gross U, Raabe A, Seifert V, et al. Active secretion of $s 100 \mathrm{~b}$ from astrocytes during metabolic stress. Neuroscience 2006;141:1697-701.

http://dx.doi.org/10.1016/j.neuroscience.2006.05.008

38. Ozawa S, Kamyia H, Tsuzuki, K. Glutamate receptors in the mammalian central nervous system. Prog Neurobiol 1998;54:581-618.

http://dx.doi.org/10.1016/S0301-0082(97)00085-3

39.Funchal C, Rosa AM, Wajner M, Wofchuk SE, Pureur RP. Reduction of Glutamate Uptake into Cerebral Cortex of Developing Rats by the BranchedChain Alpha-Keto Acids Accumulating in Maple Syrup Urine Disease. Neurochem Res 2004;29:747-53.

http://dx.doi.org/10.1023/B:NERE.0000018846.66943.30

40.Nardin P, Tramontina F, Leite MC, Tramontina AC, Quincozes-Santos A, Almeida LMV, et al. S100B content and secretion decrease in astrocytes cultured inhigh-glucose medium, Neurochem Int 2007;50:774-82.

http://dx.doi.org/10.1016/j.neuint.2007.01.013

41.Droge W. Free radicals in the physiological control of cell function Physiol Rev 2002;82:47-95.

42.Kanner BI. Glutamate transporters from brain: A novel neurotransmitter transport family. FEBS Lett 1993;325:95-9.

http://dx.doi.org/10.1016/0014-5793(93)81421-U

43.Millan MH, Chapman AG, Maldrum BS. Extracellular amino acid levels in hippocampus during pilocarpine-induced seizures. Epilepsy Res 1993;14:139-48. http://dx.doi.org/10.1016/0920-1211(93)90018-3

44.Amara SG, Fontana CK. Excitatory amino acid transporters: Keeping up with glutamate. Neurochem Int 2002;41:313-8.

http://dx.doi.org/10.1016/S0197-0186(02)00018-9

45.Dickie BGM. Neurotoxic and neurotrophic effects of chronic N-methyl$\mathrm{D}$-aspartate exposure upon mesencephalic dopaminergic neurons inorganotypic culture. Neuroscience 1996;72:731-41.

http://dx.doi.org/10.1016/0306-4522(95)00611-7

46.Ottersen OP, Storm-Mathisen J. Glutamate. in: Björklund A, Hökfelt T, Handbook of chemical neuroanatomy. Amsterdam: Elsevier, 2000, p.18.

47.Doble A. The role of excitotoxicity in neurodegenerative disease: implications for therapy. Pharmacol Ther 1999;81:163-221.

http://dx.doi.org/10.1016/S0163-7258(98)00042-4

48.Beal MF. Aging, energy, and oxidative stress in neurodegenerative diseases. Ann Neurol 1995;38:357-66.

http://dx.doi.org/10.1002/ana.410380304

49.Dugan LL, Sensi SL, Canzoniero LMT, Handran SD, Rothman S, Lin TS, et al. Mitochondrial Production of Reactive Oxygen Species in Cortical-Neurons Following Exposure to N-Methyl-D-Aspartate. J Neurosci 1995;15:6377-88.

50.Aschner M, Syversen T, Souza DO, Rocha JB, Farina M. Involvement of glutamate and reactive oxygen species in methyl mercury neurotoxicity. Braz J Med Biol Res 2007;40:285-91.

http://dx.doi.org/10.1590/S0100-879X2007000300001

51.Halliwell B, Gutteridge JM. Formation of thiobarbituric-acid-reactive substance from deoxyribose in the presence of iron salts: the role of superoxide and hydroxyl radicals. FEBS Lett 1991;128:347-52.

http://dx.doi.org/10.1016/0014-5793(81)80114-7

52.Halliwell B. Free radicals and antioxidants: A personal view. Nutr Rev 1994;52:253-65.

http://dx.doi.org/10.1111/j.1753-4887.1994.tb01453.x

53.Valko M, Leibfritz D, Moncol J, Cronin MTD, Mazur M, Telser J. Free radicals and antioxidants in normal physiological functions and human disease. 
Int J Biochem Cell Biol 2007;39:44-84.

http://dx.doi.org/10.1016/j.biocel.2006.07.001

54.Halliwell B, Gutteridge JMC. Free radical in biology and medicine. 2 ed. New York: Oxford University Press; 1989, 543p.

55.Zhu D, Tan KS, Zhang X, Sun AY, Sun GY, Lee JC. Hydrogen peroxide alters membrane and cytoskeleton properties and increases intercellular connections in astrocytes. J Cell Sci 2005;118:3695-703.

http://dx.doi.org/10.1242/jcs.02507

56. Hoozemans JJM, Veerhuis R, Rozemuller JM, Eikelenboon P. Neuroinflammation and regeneration in the early stages of Alzheimer's disease pathology. Int J Dev Neurosci 2005;24:157-65.

http://dx.doi.org/10.1016/j.ijdevneu.2005.11.001

57.Balashov KE, Comabella M, Ohashi, T, Khoury, SJ, Weiner, HL Defective regulation of IFNgamma and IL-12 by endogenous IL-10 in progressive MS. Neurology 2000;55:582-3.

58.Sulzer D. Neurodegeneration and Neuroprotection in Parkinson Disease. NeuroRx: The Journal of the American Socity for Experimental NeuroTherapeutics 2004;1:139-54.
59.Lucchinetti C, Rodriguez M. Multiple sclerosis. NEngl J Med 2000;343:938-52. http://dx.doi.org/10.1056/NEJM200009283431307

60.Bjartmar C, Trapp BD. Axonal degeneration and progessive neurologic disability in ultiple sclerosis. Neurotox Res 2003;5:157-64.

http://dx.doi.org/10.1007/BF03033380

61.Hickey W. Leucocyte traffic in the central nervous system: the participants and their roles. Semin Immunol 1999;11:125-37.

http://dx.doi.org/10.1006/smim.1999.0168

62.Karpus WJ, Ransohoff RM Chemokine regulation of experimental autoimmune encephalomyelitis: temporal and spatial expression patterns govern disease pathogenesis. J Immunol 1998;191:2667-71.

63.Huang D. Chemokines and chemokine receptors in inflammation of the nervous system: manifold roles and exquisite regulation. Immunol Rev 2000;177:52-67.

http://dx.doi.org/10.1034/j.1600-065X.2000.17709.x

64.Bacon KB, Harrison JK. Chemokines and their receptors in neurobiology: perspectives in physiology and homeostasis. J Neuroimmunol 2000;104:92-7. http://dx.doi.org/10.1016/S0165-5728(99)00266-0 